\title{
Retrieval Selectivity in the Processing of Self-referent Information: Testing the Boundaries of Self-protection
}

\author{
JEFFREY D. GREEN \\ Soka University, Aliso Viejo, California, USA \\ CONSTANTINE SEDIKIDES \\ University of Southampton, UK
}

\begin{abstract}
The mnemic neglect model (formerly the inconsistency-negativity neglect model, Sedikides \& Green, 2000) contends that people recall self-referent feedback more poorly than other-referent feedback when it carries negative implications for central self-aspects, because such feedback is perceived as threatening. We tested the prediction that participants manifest such mnemic neglect only when the central negative feedback is highly diagnostic of self-aspects (high in threat potential), not when it is low in diagnosticity (low in threat potential). Participants read negative and positive feedback behaviors that referred either to the self or another person and that were either high or low in diagnosticity. As predicted, mnemic neglect was evident only for central negative behaviors high in diagnosticity. This retrieval selectivity illustrates the strategic nature of self-protection.
\end{abstract}

People treat the self as a priceless possession: they guard it, protect it, and defend it with vigilance and vigor. They do this by denying their faults, disavowing blame for their failures, reacting angrily to direct negative feedback, recruiting additional positive qualities to offset unfavorable feedback for failure, reconstructing their past by conveniently omitting uncomplimentary details, altering the meaning of their unflattering traits to give them a flattering spin, comparing the self with less accomplished others, and withholding information likely to improve the performance of close others in domains central to the self (Baumeister, 1998; Dunning, 1999; Tesser, 2003).

We recently launched a line of research in an attempt to find out how far people will go in barricading the self from psychological threat (Sedikides, Green, \& Pinter, in press). Our primary goal was to examine nomothetically the cognitive processing and recall of individuals faced with negative self-relevant feedback. We proposed a model starting from the premise that the self-concept is a positive, well-articulated, and motivationally charged cognitive representation (Campbell \& Sedikides, 1999; Gaertner, Sedikides, Vevea, \& Iuzzini, 2002; Schwartz, 1986). So involved is this representation in information processing that any input referring to the self (e.g., "I 
would be unfaithful in an intimate relationship," "I would keep a secret") is spontaneously linked to prior self-knowledge. If the input is positive, processing advances and is elaborate. However, if the input is negative, processing is shallower and is terminated early.

More specifically, the model postulates a two-stage sequence of processing selfreferent feedback (e.g., behaviors). In Stage 1, the individual appraises the plausibility of enacting the behavior on the basis of general self-knowledge. That is, the individual compares the behavior to semantic information about the self. If the feedback is threatening to the self, processing is largely confined to Stage 1, and is relatively shallow. However, if the feedback is non-threatening to the self, processing proceeds to Stage 2, where the behavior is further compared to relevant and specific (i.e., episodic) self-knowledge.

The model also distinguishes between central and peripheral self-conceptions. The former are highly certain, self-descriptive, and important, whereas the latter are much less so (Sedikides, 1993, 1995). Negative behavioral feedback that pertains to central self-conceptions (i.e., central negative behaviors) is generally highly inconsistent with self-knowledge and highly threatening to the self. Therefore, processing of central negative behaviors is confined largely to Stage 1. For example, when considering the behavior, "I would be unfaithful in an intimate relationship," a typical individual finds a high degree of inconsistency between this behavior and the positive self-conception of being a trustworthy romantic partner. Consequently, the processing of this behavior will be shallow and central negative behaviors will be recalled poorly. In contrast, central positive behaviors are highly consistent with selfknowledge and present no threat to the self. Therefore, processing proceeds to Stage 2. For example, when considering the behavior, "I would keep a secret," a typical individual perceives a high degree of consistency between this behavior and selfknowledge. Consequently, the processing of this behavior will be deep as the individual recalls specific past examples of secret-keeping, and central positive behaviors will be recalled well.

In comparison to central negative behaviors, however, peripheral negative behaviors are far less threatening to the self (e.g., "I would show off in front of others"). Indeed, peripheral negative behaviors do not differ substantially from peripheral positive behaviors in their potential to threaten the self. Thus, these two classes of behavior will not be processed differentially (i.e., both will be processed shallowly) and, hence, no significant difference in recall is expected.

We devised a research paradigm that tests the model and, we believe, begins to probe the boundaries of self-protection (Sedikides \& Green, 2000). This paradigm allows for a direct comparison between processing and recall of both self-referent and other-referent feedback. All participants are presented with behavioral information about an individual. For half the participants, this information (i.e., feedback) pertains to the self. They are instructed to imagine that a person who knows them well has described them with a set of 32 behaviors. Half the behaviors they read are negative, half positive. Furthermore, half of the behaviors pertain to central self-aspects, half to peripheral self-aspects. For the remaining participants, the information pertains to someone else. They are instructed to imagine that a person who is very familiar with an acquaintance of theirs (Chris) has described Chris with the same set of 32 behaviors. That is, these participants also read about behaviors that are positive and negative, central and peripheral. After a brief distractor task, participants are asked to recall as many behaviors as they can. Participants display poor recall of self-referent relative to other-referent information, 
but only when the information is central and negative (e.g., "I would be unfaithful in an intimate relationship"). Participants protect the self against even the most innocuous of threats, that is, even when the threat is hypothetical. Betraying our fondness for alliteration, we have labeled this form of self-protective memory "mnemic neglect" (Sedikides \& Gregg, 2003).

We replicated the phenomenon of mnemic neglect in another, more realistic, experimental setting (Sedikides \& Green, 2000, Experiment 1). Rather than asking participants to imagine receiving behavioral feedback, we instructed them to take a computer-administered personality test and informed them that they would receive "concrete and highly accurate information about the type of person [they were]" in the form of one-sentence behaviors that they were "likely to perform." Participants in the Chris condition took the same personality test, but were led to believe that they were reading feedback about another person who had recently completed the test. The feedback consisted of the 32 behaviors described earlier. The recall pattern also mimicked that described earlier. Recall for central negative self-referent information was lower than recall for central positive self-referent information as well as central negative Chris-referent information (i.e., the very same behaviors that described someone else). Thus, mnemic neglect was evident both in a hypothetical setting and in a realistic feedback setting.

According to the model, central negative self-referent behaviors pose a threat to the self and that is why they are processed minimally. Another experiment (Sedikides \& Green, 2000, Experiment 3) directly tested this notion. Behaviors were presented one at a time on a computer screen. The experiment manipulated behavior presentation time: Half of the participants had only limited time (i.e., two seconds) to process each behavior, whereas the remaining half had ample time (i.e., eight seconds). We maintained that, if an important reason for the relatively poor recall of central negative self-referent behaviors is the motivated allocation of minimal processing time to these behaviors, then minimizing the processing time available for all behaviors would result in equally poor recall across the board. Consequently, we expected for central negative behaviors to be recalled as poorly as all other classes of behaviors in the limited processing time conditions. However, we expected for the phenomenon of mnemic neglect to replicate in the ample processing time condition, as this condition was analogous to that of previous experiments. Our hypotheses were confirmed.

In follow-up research (Sedikides \& Green, in press), we attempted to disentangle the effects of information inconsistency and information valence. Is the phenomenon of mnemic neglect due to feedback being inconsistent with the self or being negative? We identified through pilot testing individuals who considered themselves relatively untrustworthy or unkind as well as individuals who considered themselves trustworthy or kind. In a separate session one week later, these participants processed and then recalled the same untrustworthy/trustworthy and unkind/kind behaviors used in previous experiments. Participants with both positive and negative self-conceptions recalled negative behaviors poorly relative to positive behaviors. Stated otherwise, both untrustworthy and trustworthy participants recalled poorly untrustworthy behaviors, and both unkind and kind participants recalled poorly unkind behaviors. Thus, the negativity of the behavioral feedback rather than its inconsistency accounts for mnemic neglect. (For a conceptually similar finding, see Sedikides, 1993, Experiment 4.) Consequently, we will refer to our theoretical model as the mnemic neglect model. ${ }^{1}$ 


\section{The Present Research}

Our past research has demonstrated that individuals protect the self by processing self-referent information strategically and selectively. This claim relies on the nature of observed recall patterns: poor recall for self-referent feedback is manifested only when this feedback is negative and pertains to central self-aspects. However, what are the boundaries of retrieval selectivity? Do individuals manifest mnemic neglect for central negative behaviors across the board? Is self-protective memory indiscriminate for negativity of all sorts? Or, alternatively, does it matter whether the behavioral feedback is high or low in diagnosticity? This last question is addressed in the present investigation.

Behavioral diagnosticity refers to the extent to which a behavior is indicative of an underlying trait. Good indicators (e.g., "I would be unfaithful when in an intimate relationship") for a trait (e.g., untrustworthy) are termed high diagnosticity behaviors, whereas poor trait indicators (e.g., "I would take a pen from the bank after signing a check") are termed low diagnosticity behaviors. In the case of negative (especially central negative) self-aspects, high diagnosticity indicators are more self-threatening than their low diagnosticity counterparts. For instance, a person would be more threatened when told that she/he is the kind of person who "would be unfaithful in an intimate relationship" than the kind of person who "would take a pen from the bank after signing a check."

In our previous research (Sedikides \& Green, 2000, in press), we used exclusively high diagnosticity behaviors. However, this tactic resulted in a rather incomplete test of the mnemic neglect model. A more comprehensive and compelling test would have required inclusion of both high and low diagnosticity behaviors. Is self-protection so pervasive and automatic that negative behaviors are processed minimally regardless of their implications for underlying traits? The model is predicated on the tenet that memorial self-protection, being strategic, is observed under high, but not low, selfthreat. Hence, the model predicts that recall for central negative self-referent behavioral feedback will be attenuated (as in previous experiments) when the feedback is high diagnosticity, but not (in contrast to previous experiments) when the feedback is low diagnosticity. Such a pattern would represent a more complete and rigorous test of the model, would clarify further the construct of mnemic neglect, and would provide a fuller picture of the strategic nature of self-protection.

\section{Method}

\section{Pilot Study}

We began by conducting a pilot study the purpose of which was the selection of high and low diagnosticity behaviors for use in the main experiment. We pilot tested both the high diagnosticity behaviors employed in previous research (Sedikides \& Green, 2000) and the low diagnosticity behaviors that we generated for the purposes of the current investigation (see Appendix). Seventy-eight University of North Carolina at Chapel Hill (UNC-CH) undergraduate students participated in the study in fulfillment of an introductory psychology course option. The behaviors were blocked by trait dimension (e.g., unkind - kind) and were randomly ordered on each page. The instructions read: "Please rate each of the following behaviors on how informative of the trait [trustworthy] you think it is. Would performing the behavior tell you a great deal about how [trustworthy] a person is (informative), or very little 
about how [trustworthy] a person is (uninformative)?" Participants rated each behavior on a nine-point scale, bounded by the endpoints 1 (extremely uninformative of the trait) and 9 (extremely informative of the trait). The results, presented in Table 1 , confirmed that participants perceived high diagnosticity behaviors $(M=7.15)$ as more informative of the respective traits than low diagnosticity behaviors $(M=5.74), t(77)=11.59, p<.001$. We obtained analogous results when we conducted separate analyses for each behavioral set.

\section{Participants and Experimental Design}

Participants in the main experiment were $255 \mathrm{UNC}-\mathrm{CH}$ introductory psychology student volunteers, tested in groups of 2-6. Participants were unable to see each other when seated in individual carrels. We used a 2 (Referent: self, Chris) $\times 2$ (Behavior Diagnosticity: high diagnostic behaviors, low diagnostic behaviors) $\times 2$ (Behavior Type Order: central behaviors presented first, peripheral behaviors presented first) $\times 2$ (Behavior Valence Order: positive behavior first, negative behavior first) $\times 2$ (Behavior Type: central behaviors, peripheral behaviors $) \times 2$ (Behavior Valence: positive behaviors, negative behaviors) factorial design, in which the first four factors were between-subjects and the last two factors were withinsubjects.

\section{Procedure}

Participants read four pages of behaviors exemplifying four trait dimensions. Each page pertained to a single trait dimension (e.g., untrustworthy-trustworthy) and contained eight behaviors. Participants read the 32 behaviors under different instructional sets. Those in the self condition considered the behaviors with reference to the self. Specifically, they were asked to "consider the following description of YOURSELF. Think of the description as being based on actual knowledge of people who know YOU well. Think of the description as real." Participants in the Chris condition considered "a description of a person named CHRIS. Think of the description as being based on actual knowledge of people who know CHRIS well. Think of the description as real." This manipulation constituted the referent factor.

The four dimensions were trustworthy-untrustworthy, kind-unkind, modest-immodest, and uncomplaining-complaining. In previous research, the first two trait dimensions were rated as more central by undergraduate students, whereas the last two dimensions were rated as more peripheral (Sedikides,

TABLE 1 Behavior Diagnosticity Means and $t$-values in Pilot Study

\begin{tabular}{lccrc}
\hline Trait dimension & $\begin{array}{c}\text { High } \\
\text { diagnosticity } \\
\text { behaviors }\end{array}$ & $\begin{array}{c}\text { Low } \\
\text { diagnosticity } \\
\text { behaviors }\end{array}$ & $t(77)$ & $p$ \\
\hline Untrustworthy - trustworthy & 7.50 & 5.28 & 14.52 & .001 \\
Unkind - kind & 7.33 & 6.10 & 9.15 & .001 \\
Complaining- uncomplaining & 6.79 & 5.66 & 6.83 & .001 \\
Immodest-modest & 7.00 & 5.92 & 7.26 & .001 \\
\hline
\end{tabular}


1993). In addition, pilot test ratings validated the behaviors as central or peripheral (Sedikides \& Green, 2000). This manipulation constituted the behavior type factor (central or peripheral). Four positive and four negative behaviors represented each trait dimension. This manipulation constituted the behavior valence factor (positive or negative). Participants read either the high diagnosticity behavior set or the low diagnosticity behavior set. This was the diagnosticity factor.

As in previous research (e.g., Sedikides \& Green, 2000), we used two between-subjects order variables. We randomized the valence order of the behaviors for each trait dimension under the stipulation that no more than two same-valenced behaviors appear sequentially. One randomization pattern (i.e., negative, positive, negative, positive, negative, negative, positive, positive) was presented to half of participants, whereas the alternative mirror-image randomization pattern (i.e., positive, negative, positive, negative, positive, positive, negative, negative) was presented to the remaining half of participants. The inclusion of this factor (behavior valence order) was intended to control for recency and primacy effects. Another control factor (behavior type order) involved presentation order of central versus peripheral behaviors. Half of the participants read the central behaviors first, whereas the other half read the peripheral behaviors first.

Participants read through the booklet of behaviors at their own pace. Subsequently, they completed a distractor task, which consisted of listing as many of the United States as possible in two and a half minutes, followed by a surprise recall task. Participants wrote down the behaviors in a booklet of blank sheets of paper. They were instructed: (1) to write down one behavior per sheet in any order that the behaviors came to mind; (2) not to turn back to previous pages; and (3) to try to be as accurate as possible without worrying about recalling the behaviors verbatim. The recall task lasted five minutes. A debriefing concluded the experimental session.

\section{Results}

Two independent judges coded the free recall data according to a general meaning or "gist" criterion, as in our previous research (Sedikides \& Green, 2000 , in press). The coders agreed on over $96 \%$ of the recalled items and resolved discrepancies through discussion. Intrusions (e.g., recalling a behavior that was not presented, writing the same behavior twice, or changing the valence of a recalled behavior) constituted $3.7 \%$ of the recalled items and were removed prior to data analysis. This percentage is comparable to low intrusion rates reported in past relevant research (Sedikides \& Green, 2000, in press). Also in line with this past research, we enforced an inclusion rule of no more than three intrusions per participant; this rule led to the exclusion of data from two participants. We discarded data from another eight participants, because they misunderstood the recall instructions and listed traits instead of behaviors. Thus, the final number of participants entered in the data analyses was 245. (The cell sizes ranged from 13-16 participants.) Table 2 contains the proportion of behaviors recalled as a function of referent, behavior type, behavior valence, and behavior diagnosticity.

The most critical source of support for the mnemic neglect model has been the triple interaction among referent, behavior type, and behavior valence (Sedikides \& 
TABLE 2 Mean Proportion of Behaviors Recalled as a Function of Referent, Behavior Type, Behavior Valence, and Behavior Diagnosticity

\begin{tabular}{|c|c|c|c|c|}
\hline & \multicolumn{2}{|c|}{ Central behaviors } & \multicolumn{2}{|c|}{ Peripheral behaviors } \\
\hline & Negative & Positive & Negative & Positive \\
\hline \multicolumn{5}{|l|}{ High diagnosticity } \\
\hline Self-referent & .29 & .46 & .24 & .21 \\
\hline Chris-referent & .41 & .43 & .20 & .21 \\
\hline \multicolumn{5}{|l|}{ Low diagnosticity } \\
\hline Self-referent & .30 & .28 & .33 & .28 \\
\hline Chris-referent & .36 & .32 & .38 & .35 \\
\hline
\end{tabular}

Green, 2000). We replicated this finding, $F(1,229)=6.15, p<.02$. As in our past research, we broke down this effect by examining the Referent $\times$ Behavior Valence interaction separately for central and peripheral behaviors. In the case of central behaviors, the Referent $\times$ Behavior Valence interaction was significant, $F(1$, $240)=6.19, p<.01$. Participants who engaged in self-referent processing recalled fewer central negative behaviors $(M=.30)$ than those who engaged in Chrisreferent processing $(M=.39), t(240)=3.62, p<.001$. In addition, participants who engaged in self-referent processing recalled fewer central negative than central positive $(M=.37)$ behaviors, $t(123)=9.95, p<.002$. However, in the case of peripheral behaviors, the Referent $\times$ Behavior Valence interaction was not significant, $F(1,240)=0.83, p<.36$.

Importantly, the above triple interaction was qualified by behavior diagnosticity, $F(1,229)=4.67, p<.03$. To explicate this quadruple interaction, we examined the Referent $\times$ Behavior Type $\times$ Behavior Valence interaction separately for high diagnosticity and low diagnosticity behaviors.

In the case of high diagnosticity behaviors, the Referent $\times$ Behavior Type $\times$ Behavior Valence interaction was significant, $F(1,117)=10.51, p<.002$. We further decomposed this interaction by examining the Referent $\times$ Behavior Valence interaction separately for central and peripheral behaviors. With regard to central behaviors, the Referent $\times$ Behavior Valence interaction was significant, $F(1,117)=13.05, p<.001$. Participants who engaged in selfreferent processing manifested lower recall for central negative $(M=.29)$ than central positive $(M=.46)$ behaviors, $t(63)=5.23, p<.001$. That is, participants who processed high diagnosticity behaviors self-referentially retrieved selectively (i.e., poorly) the most threatening information. In addition, participants who engaged in self-referent processing $(M=.29)$ recalled fewer central negative behaviors than those who engaged in Chris-referent processing $(M=.41)$, $t(123)=-3.80, p<.001$. Stated somewhat differently, participants retrieved selectively (i.e., poorly) the very same central negative behaviors when these behaviors referred to the self relative to Chris. With regard to peripheral behaviors, the Referent $\times$ Behavior Valence interaction was not significant, $F(1$, $117)=0.67, p<.42$. Peripheral negative self-referent behaviors do not pose a direct threat to the self and are thus not recalled poorly compared to either peripheral positive self-referent behaviors or peripheral negative Chris-referent behaviors. 
Low diagnosticity behaviors do not pose a serious threat to the self either. Therefore, no disproportionately low recall of central negative behaviors will be observed. Indeed, in the case of low diagnosticity behaviors, the triple interaction among referent, behavior type, and behavior valence was not significant, $F(1$, $112)=0.05, p<.82$. Of course, the Referent $\times$ Behavior Valence interactions for central behaviors, $F(1,112)=0.02, p<.88$, and peripheral behaviors, $F(1$, $112)=0.24, \quad p<.62$, were not significant either. As another illustration, participants' recall for central negative $(M=.30)$ and central positive $(M=.28)$ self-referent behaviors was not significantly different, $t(59)=-0.82, p<.42$. Finally, recall of central negative behaviors did not differ significantly as a function of whether participants processed these behaviors with reference to the self $(M=.30)$ or Chris $(M=.36), t(118)=-1.21, p<.23$.

\section{Supplementary Findings}

Some ambiguities may arise with regard to the interpretation of the four-way interaction (Table 2). If one examines recall only for central negative self-referent behaviors, one might question whether mnemic neglect occurred in the high diagnosticity condition $(M=.29)$ when recall of low diagnosticity behaviors was essentially equal $(M=.30)$. However, we believe that the phenomenon of mnemic neglect must be appraised against the backdrop of the entire design. Simply comparing the means for the high and low diagnosticity cells is misleading due to additional observed differences between the two behavior sets. Overall, participants in the high diagnosticity conditions recalled approximately equal proportions of behaviors as participants in the low diagnosticity conditions, $F(1,229)=1.74, p$ $<.19$. However, the behavior type by diagnosticity interaction was significant, $F(1$, $229)=85.38, p<.001$. Replicating previous research, participants who processed high diagnosticity behaviors recalled significantly more central behaviors $(M=.40)$ than peripheral behaviors $(M=.22), t(124)=10.37, p<.001$. In contrast, participants who processed low diagnosticity behaviors recalled marginally more peripheral behaviors $(M=.34)$ than central behaviors $(M=.31), t(119)=-1.74$, $p<.085$.

Interestingly, $t$-tests that further examine this effect according to each behavior class (i.e., central positive, central negative, peripheral positive, peripheral negative) are quite revealing. Both self-referent participants, $t(122)=5.43, p<.001$, and Chris-referent participants, $t(119)=3.55, p<.001$, recalled more high than low diagnosticity central positive behaviors. Also, both self-referent participants, $t(122)=-2.23, p<.03$, and Chris-referent participants, $t(119)=-5.30, p<.001$, recalled fewer high than low diagnosticity peripheral positive behaviors. Additionally, both self-referent participants, $t(122)=-2.95, p<.001$, and Chris-referent participants, $t(119)=-6.10, p<.001$, recalled fewer high than low diagnosticity peripheral negative behaviors. The only exception to this general pattern is central negative behaviors: Chris-referent participants recalled significantly more high than low diagnosticity behaviors, $t(119)=2.48, p<.02$, but self-referent participants displayed approximately equal recall of high diagnosticity and low diagnosticity behaviors, $t(122)=-0.35, p<.73$. In summary, we think that the mnemic neglect effect ought to be considered in light of this central versus peripheral difference between the two stimulus material sets. Our data analytic strategy began with examining the full model (i.e., the four-way interaction) and breaking down the model accordingly in order to explain this higher-order interaction. 


\section{Discussion}

According to the mnemic neglect model, central negative self-referent behaviors present a threat to the self. Hence, participants will process them shallowly and recall them poorly. Indeed, as our past research (Sedikides \& Green, 2000, in press) demonstrated, participants recall central negative self-referent behaviors poorly relative to both central positive self-referent behaviors and to central negative otherreferent behaviors. Self-protective memory is selective and strategic.

However, all the behaviors used in our previous research were highly diagnostic or indicative of self-aspects. As such, behavior diagnosticity may narrow the scope of mnemic neglect. The question arises: is the phenomenon of mnemic neglect observed across all central negative self-referent behaviors or, instead, is it restricted to the subset of these behaviors high in diagnosticity? High diagnosticity behaviors reveal clearly the character of the person who performed them, whereas low diagnosticity behaviors are imbued with ambiguity regarding the character of the performer.

In the present experiment, we ventured a more complete, rigorous, and compelling test of the mnemic neglect model, exploring the strategic and selective nature of self-protection. In particular, we built on the design of our previous experiments (i.e., Sedikides \& Green, 2000, Experiments 1-2), but additionally manipulated behavior diagnosticity directly. We reasoned that, if the perception of threat drives mnemic neglect, then this phenomenon will be present for high diagnosticity behaviors, but absent for low diagnosticity behaviors. The results confirmed our hypothesis, thus delineating some informative boundary conditions of the phenomenon of mnemic neglect.

A natural confound may exist between behavior valence and behavior diagnosticity. Negative high diagnosticity behaviors may be viewed as more unfavorable than negative low diagnosticity behaviors, whereas positive high diagnosticity behaviors may be viewed as more favorable than positive low diagnosticity behaviors. We believe, however, that this possible confound does not call the interpretation of our findings into question, because we included a control group. That is, we used the same behaviors for both the experimental (self) and the control (Chris) group. Given that the behaviors were identical, observed differences in recall can only be attributed to the behavioral referent.

At the same time, we do not wish to claim that self-protection is the only selfevaluation motive. Several other self-evaluation motives have been identified and empirically tested (Sedikides \& Strube, 1997). One of them is self-improvement (Pemberton \& Sedikides, 2001). A person for whom the self-improvement motive is chronically or contextually activated might recall more central negative self-referent feedback, especially when the feedback refers to modifiable self-attributes (Dauenheimer, Stahlberg, Spreeman, \& Sedikides, 2002). Future research will need to address this issue by examining whether the feedback pertains to modifiable versus unmodifiable self-attributes. Future research also might delve more deeply into the exact processing locus of mnemic neglect. Central negative information about the self is weakly encoded (Sedikides \& Green, 2000, Experiment 3), but also such information may be blocked during retrieval. If so, what are the mechanisms through which this information is blocked?

In conclusion, the present findings illustrate the strategic and selective nature of self-protection. Individuals do not pour psychological resources indiscriminately into protecting the self from external threat. In particular, individuals do not self- 
protect when their peripheral self-conceptions are under threat, either unequivocal (i.e., high diagnosticity feedback) or equivocal (i.e., low diagnosticity feedback), nor do they self-protect when their central self-conceptions are under merely equivocal threat. In contrast, individuals self-protect with vigilance and vigor when their central self-conceptions are under unequivocal threat. In this case, individuals selfprotect even against hypothetical psychological danger. This rather judicious and selective use of cognitive resources signifies that people consider the self-concept as a precious and cherished possession - a consideration that is justified given the functionality of the self-system (Mischel \& Morf, 2003; Sedikides \& Skowronski, 2003).

\section{Note}

1. Initially we labeled our model the "inconsistency-negativity neglect model" (Sedikides \& Green, 2000).

\section{References}

Baumeister, R. F. (1998). The self. In D. T. Gilbert, S. T. Fiske, \& G. Lindzey (Eds.), The handbook of social psychology (Vol. 1, pp. 680-740). New York: Oxford University Press.

Campbell, W. K., \& Sedikides, C. (1999). Self-threat magnifies the self-serving bias: A metaanalytic integration. Review of General Psychology, 3, 23-43.

Dauenheimer, D. G., Stahlberg, D., Spreeman, S., \& Sedikides, C. (2002). Self-enhancement, self-assessment, or self-verification? The intricate role of trait modifiability in the selfevaluation process. Revue Internationale De Psychologie Sociale, 15, 89-112.

Dunning, D. (1999). A newer look: Motivated social cognition and the schematic representation of social concepts. Psychological Inquiry, 10, 1-11.

Gaertner, L., Sedikides, C., Vevea, J., \& Iuzzini, J. (2002). The "I," the "We," and the "When:" A meta-analysis of motivational primacy in self-definition. Journal of Personality and Social Psychology, 83, 574-591.

Mischel, W., \& Morf, C. M. (2003). The self as a psycho-social dynamic processing system: A meta-perspective on a century of the self in psychology. In M. R. Leary \& J. P. Tangney (Eds.), Handbook of self and identity (pp. 15-43). New York, NY: Guilford.

Pemberton, M., \& Sedikides, C. (2001). When do individuals help close others improve? Extending the self-evaluation maintenance model to future comparisons. Journal of Personality and Social Psychology, 81, 234-246.

Schwartz, R. M. (1986). The internal dialogue: On the asymmetry between positive and negative coping thoughts. Cognitive Therapy and Research, 10, 591-605.

Sedikides, C. (1993). Assessment, enhancement, and verification determinants of the selfevaluation process. Journal of Personality and Social Psychology, 65, 317-338.

Sedikides, C. (1995). Central and peripheral self-conceptions are differentially influenced by mood: Tests of the differential sensitivity hypothesis. Journal of Personality and Social Psychology, 69, 759-777.

Sedikides, C., \& Green, J. D. (2000). On the self-protective nature of inconsistency-negativity management: Using the person memory paradigm to examine self-referent memory. Journal of Personality and Social Psychology, 79, 906-922.

Sedikides, C., \& Green, J. D. (in press). What I don't recall can't hurt me: Information negativity versus information inconsistency as determinants of memorial self-defense. Social Cognition.

Sedikides, C., Green, J. D., \& Pinter, B. T. (in press). Self-protective memory. In D. Beike, J. Lampinen, \& D. Behrend (Eds.), Memory and the self. Philadelphia, PA: Psychology Press. 
Sedikides, C., \& Gregg, A. P. (2003). Portraits of the self. In M. A. Hogg \& J. Cooper (Eds.), Sage handbook of social psychology (pp. 110-138). London: Sage Publications.

Sedikides, C., \& Skowronski, J. J. (2003). Evolution of the self: Issues and prospects. In M. R. Leary \& J. P. Tangney (Eds.), Handbook of self and identity (pp. 594-609). New York: Guilford.

Sedikides, C., \& Strube, M. J. (1997). Self-evaluation: To thine own self be good, to thine own self be sure, to thine own self be true, and to thine own self be better. In M. P. Zanna (Ed.), Advances in experimental social psychology (Vol. 29, pp. 209-269). New York: Academic Press.

Tesser, A. (2003). Self-evaluation. In M. R. Leary \& J. P. Tangney (Eds.), Handbook of self and identity (pp. 275-290). New York: Guilford Press.

\section{Appendix-Behaviors Used in the Experiment}

\section{High Diagnosticity Behaviors}

\section{Trustworthy-Untrustworthy}

$\mathrm{X}$ would borrow other people's belongings without their knowledge. (6.00)

$\mathrm{X}$ would keep secrets when asked to. (8.77)

$\mathrm{X}$ would be unfaithful when in an intimate relationship. (7.24)

$\mathrm{X}$ would follow through on a promise made to friends. (8.56)

X would often lie to X's parents. (6.62)

An employer would not rely on $\mathrm{X}$ to have an important project completed by the deadline. (6.53)

A teacher would leave $\mathrm{X}$ alone in a room while taking a test and not be afraid that $\mathrm{X}$ would cheat. (8.41)

People would be willing to tell $\mathrm{X}$ embarrassing things about themselves in confidence. (7.86)

\section{Kind-Unkind}

$\mathrm{X}$ would make fun of others because of their looks. (6.27)

$\mathrm{X}$ would offer to care for a neighbor's child when the baby-sitter couldn't come. (7.76)

$\mathrm{X}$ would purposely hurt someone to benefit X. (6.92)

$\mathrm{X}$ would help people by opening a door if their hands were full. (7.89)

$\mathrm{X}$ would refuse to lend class notes to a friend who was ill. (6.76)

$\mathrm{X}$ would make an obscene gesture to an old lady. (6.55)

$\mathrm{X}$ would help a handicapped neighbor paint his or her house. (8.30)

$\mathrm{X}$ would volunteer time to work as a big brother or big sister to a child in need. (8.32)

\section{Uncomplaining-Complaining}

$\mathrm{X}$ would look for faults even if X's life was going well. (6.51)

$\mathrm{X}$ would rarely inform others about physical ailments. (7.04)

When $\mathrm{X}$ would not like to do something, $\mathrm{X}$ would constantly mention it. (6.55)

$\mathrm{X}$ would overlook the bad points about a roommate. (7.21)

$\mathrm{X}$ would constantly talk about how much stuff there is to be done. (6.28)

$\mathrm{X}$ would pick only the bad points to describe the classes $\mathrm{X}$ attends. (6.30)

$\mathrm{X}$ would minimize bad experiences when telling about them. (6.90)

$\mathrm{X}$ would tolerate situations even when not having a good time. (7.56)

\section{Modest-Immodest}

$\mathrm{X}$ would act in a condescending manner to other people. (5.68)

$X$ would take the focus off $X$ and redirect it to others. (7.64)

$\mathrm{X}$ would point out others' weaknesses to make X look better. (6.26)

$\mathrm{X}$ would let some of X's achievements go by unaccredited. (7.96)

$\mathrm{X}$ would talk more about $\mathrm{X}$ than about others. (6.56)

$\mathrm{X}$ would show off in front of others. (6.42) 
$\mathrm{X}$ would give others the credit for a group success. (7.65)

$\mathrm{X}$ would never openly brag about X's accomplishments. (7.84)

\section{Low Diagnosticity Behaviors}

\section{Trustworthy-Untrustworthy}

$\mathrm{X}$ would use the toothpaste of a roommate without asking. (5.22)

$\mathrm{X}$ would not ask a friend for help when writing a paper for a class. (5.69)

$\mathrm{X}$ would take a pen from the bank after signing a check. (4.96)

$\mathrm{X}$ would promptly pick up a friend at the agreed-upon time. (4.55)

$X$ would underestimate the number of copies made at a copy center. (4.42)

$\mathrm{X}$ would forget for a week to return a borrowed book to a friend. (5.15)

$\mathrm{X}$ would be asked to plan a surprise party for a friend. (6.91)

$\mathrm{X}$ would remember to communicate a phone message. (5.30)

\section{Kind-Unkind}

$\mathrm{X}$ would chuckle when someone slid down an icy hill. (4.92)

$\mathrm{X}$ would compliment a friend who was dressed very nicely. (7.40)

$\mathrm{X}$ would ask for a refund for very slow service at a restaurant. (6.54)

$\mathrm{X}$ would take a friend out for a beer to celebrate the friend's graduation. (5.51)

$\mathrm{X}$ would forget family members' birthdays. (7.18)

$X$ would avoid an old flame at a party if there had been a bad break-up. (5.14)

$\mathrm{X}$ would oil a squeaky door in the dorm hallway. (6.59)

$\mathrm{X}$ would return a greeting when a stranger says hello on the street. (5.46)

\section{Uncomplaining-Complaining}

$\mathrm{X}$ would complain about extremely cold or wet weather. (6.06)

$\mathrm{X}$ would talk badly about the current politicians. (4.71)

$\mathrm{X}$ would say nothing when someone unknowingly cut in line at the grocery store. (5.86)

$\mathrm{X}$ would criticize a terrible movie while watching it in the theater. (6.06)

$\mathrm{X}$ would acknowledge an apology if someone stepped on X's foot. (4.96)

$\mathrm{X}$ would get irritated at weather changes. (6.81)

$\mathrm{X}$ would not be bothered when other people whine. (6.46)

$\mathrm{X}$ would not send back restaurant food that was slightly overcooked. (4.31)

\section{Modest-Immodest}

$\mathrm{X}$ would become rowdy when X's favorite team won. (5.01)

$\mathrm{X}$ would give a homeless man some spare change but not draw attention to it. (5.99)

$\mathrm{X}$ would tell friends after getting a high grade on a test. (6.57)

$\mathrm{X}$ would consider $\mathrm{X}$ only as good-looking as the average person. (7.22)

$X$ would openly celebrate a victory in a sport. (4.65)

$\mathrm{X}$ would brag about a sibling's acceptance to Harvard. (6.57)

$\mathrm{X}$ would not mention meeting a famous celebrity. (6.17)

$\mathrm{X}$ would not overemphasize X's strong points on a graduate school application. (5.17)

\section{Notes}

1. "X" refers to either self (i.e., "I") or Chris.

2. Numbers in parentheses are the diagnosticity pilot study means.

3. Participants rated each behavior on a nine-point scale, with the endpoints being 1 (extremely uninformative of the trait) and 9 (extremely informative of the trait). 